\title{
Los vehículos motorizados privados y el problema de transporte público en los centros históricos: el caso de Cuenca-Ecuador ${ }^{1}$
}

\author{
Private motor vehicles and the problem of public transport in \\ the historic centres: the case of Cuenca-Ecuador
}

Por:

María Soledad Moscoso

Cordero

Universidad de Cuenca

Recibido: 15 de febrero 2012 Aceptado: 15 de Marzo 2012

\section{Resumen:}

El transporte, la peatonalización y la conservación son tres principales problemas en la gestión de las ciudades patrimoniales, especialmente en las ciudades latinoamericanas, donde los recursos económicos limitan su acceso a sistemas sostenibles de transporte.

Después de contar con un panorama general del problema y sus causas, se han investigado las soluciones así como la implementación de políticas adecuadas, vinculadas con el tema, en los centros históricos. Asimismo, se analizaron casos exitosos concretos de los sistemas de gestión del tráfico desarrollados en ciudades del Patrimonio Mundial con la finalidad de encontrar soluciones transferibles y adaptables al contexto de Cuenca, una de las dos ciudades Patrimonio de la Humanidad del Ecuador.

Palabras clave: Tranporte público, sostenibilidad, centro histórico, Cuenca, Ecuador.

\section{Abstract:}

Transport, pedestrianization and conservation are three main problems in the management of heritage cities, especially in the Latin American cities, where economic resources limit their access to sustainable transport systems.

After having an overview of the problem and its causes, have been investigated solutions as well as the implementation of appropriate policies, related to the subject, in the historical centres. Likewise, we have analyzed successful cases of traffic management systems developed in cities of the world heritage in order to find solutions transferable and that they can be adapted to the context of Cuenca, one of the two cities patrimony of the humanity of the Ecuador.

Keywords: Public transport, sustainability, historical centre, Cuenca, Ecuador. 


\section{Introducción:}

El objetivo de este trabajo de investigación es comprender de una manera más profunda el problema generado por el intenso tráfico en ciudades patrimoniales, y específicamente en el Centro Histórico de Cuenca (Ecuador). Un análisis de las causas y los efectos será necesario para identificar buenas prácticas y exitosas políticas de gestión del tráfico.

La necesidad de una mejor gestión del tráfico en Cuenca está motivada por el acelerado incremento del número de vehículos en relación con la densidad de población. En la actualidad, este problema ha generado un renovado interés del Gobierno local a fin de encontrar soluciones precisas para tomar las decisiones importantes nunca antes tomadas. Por lo tanto, es de vital importancia y, de hecho necesario, buscar posibles soluciones con respecto a la gestión del tráfico desde el punto de vista de la conservación patrimonial.

Dado que la congestión vehicular es un problema recurrente en muchas ciudades históricas, creemos que es necesario encontrar en ellas algunas buenas prácticas que pueden ser transmitidas a otras ciudades y que, de preferencia, no requieran de una elevada inversión, con el fin de mantener la calidad ambiental en las de alto valor histórico-arquitectónico.

Entre la bibliografía pertinente analizada, adicional a la información técnica local producida por la Municipalidad de Cuenca sobre el tránsito y el transporte, podemos citar el libro editado por Robert Pickard, Management of Historic Centres, que contiene varios ejemplos de gestión de centros históricos. Cada uno de los casos estudiados cuenta con una descripción completa de las políticas y sistemas implantados para gestionar sus problemas de tráfico, mientras cuidan su patrimonio edificado. También es interesante echar un vistazo a casos como los referidos por Javier Gutiérrez Puebla en su artículo "Transporte, movilidad y turismo en los centros históricos", el mismo que contiene ejemplos de útiles políticas para la adecuada gestión del tránsito en los centros históricos hispanos.
Se ha revisado también bibliografía descriptiva sobre la tecnologías de sistemas de transporte, entre ellos: una tesis de maestría escrita por Bjorn Gunnarson y Andreas Lofgren, de la Universidad Técnica de Lulea, relacionada con las experiencias de la implementación del tranvía en varias ciudades europeas; un artículo de Marion Edwards y Roger L. Mackett, que describe las ventajas y desventajas de los diferentes tipos de sistemas de transporte urbano, entre otros.

Este trabajo de investigación se llevó a cabo utilizando un método exploratorio y descriptivo, mediante el análisis de la información bibliográfica pertinente, que también incluye revistas académicas y artículos en periódicos locales, en los que se expresa la opinión pública sobre el tema. Después de un examen crítico y comparativo de la situación fue posible proponer soluciones adecuadas al asunto de la proliferación de vehículos motorizados privados y la deficiente transportación pública, a nivel de políticas de gestión.

Como es obvio, pero nunca se insistirá lo suficiente: "Si se planifica ciudades para los coches y el tráfico, se consigue coches y tráfico. Si se planifica para las personas y los lugares se consigue gente y lugares"(F. Kent, 2005).

\section{El problema del tráfico en las ciudades patrimoniales}

El excesivo número de vehículos circulando por las estrechas calles de los centros históricos es un problema significativo y repetitivo en muchas ciudades históricas, incluidas o no en la lista de Patrimonio Cultural de la Organización de las Naciones Unidas para la Educación, la Ciencia y la Cultura (UNESCO). Este es un tema que no está relacionado con la capacidad económica de las ciudades (Ver Fotografía $\mathrm{N}^{\circ} .1$ ), porque la riqueza no garantiza una buena gestión de zonas tan delicadas, donde la mínima complicación puede causar grandes dificultades para su conservación.

Hay dos clases de efectos producidos por el intenso tráfico en las ciudades patrimoniales, uno de ellos tiene que ver con los daños físicos, y el otro con el deterioro de la habitabilidad de la Ciudad Histórica. En el primer caso, los contaminantes procedentes del motor de transporte causan daños físicos a los edificios históricos por la acción de los óxidos de nitrógeno, carbono y azufre desprendidos de la combustión de hidrocarburos; estos compuestos actúan como un catalizador que intensifica la acción química de agua ${ }^{2}$. Entre los daños físicos producidos en los edificios históricos están considerados problemas como: exfoliación de pintura, costras oscuras, desprendimientos por capas, entre otros. Otro peligroso efecto producido por la circula- 
Los vehículos motorizados privados y el problema del transporte público...

ción de vehículos pesados de motor es la vibración que afecta a los débiles materiales de construcción y estructuras de edificios patrimoniales produciéndose grietas en sus paredes.

Las ciudades históricas, todos los días, están sometidas a una mezcla explosiva de tráfico de vehículos, peatones, compradores y trabajadores. Toda esta presión puede afectar el sentido de pertenencia de sus habitantes y visitantes y, finalmente, al espíritu del lugar. ${ }^{3}$

Las calles de los centros históricos nunca fueron previstas para ser transitadas por coches y/o autobuses, al menos no de la forma intensiva como se da en la actualidad. La creciente concentración de actividades terciarias en el núcleo central ha convertido a varios edificios históricos en oficinas, tiendas, etc. El desarrollo de éstas demanda plazas de aparcamiento que, por supuesto, tampoco estuvo previsto albergarlos; así, las calles se han convertido en espacios de estacionamiento público, generando una reducción de la capacidad de las vías que afecta a la movilidad en la ciudad. A nivel mundial, la falta de buenas políticas y la implementación de servicios de transporte en función de la vulnerabilidad de las ciudades históricas han generado tensiones en los centros históricos de todo el mundo.

Por lo tanto, la gestión de la accesibilidad y la movilidad en las áreas históricas tiene que ser tratada con cuidado. Es necesario crear un plan de manejo adecuado de cada ciudad histórica con el fin de evitar la implementación de políticas de transporte demasiado restrictivas que también podrían afectar a la habitabilidad, expulsando a sus habitantes y a largo plazo afectando a su conservación (Gutiérrez Puebla, 1998: 242-245).

La experiencia demuestra que el control del tráfico y las estrategias de regulación de estacionamiento han sido en un principio vistos con malos ojos por los habitantes de los centros históricos; sin embargo, una vez que los buenos resultados comienzan a ser evidentes, son bien aceptados por las comunidades.

\section{Gestión del tráfico en los cascos históricos}

Cuando se trabaja en una ciudad histórica no es posible aplicar una "receta", sin considerar sus particularidades, como si se tratara de un lienzo en blanco; por el contrario, es imprescindible realizar un profundo análisis que dará lugar a la toma de decisiones bien informadas. No obstante, es aconsejable informarse de

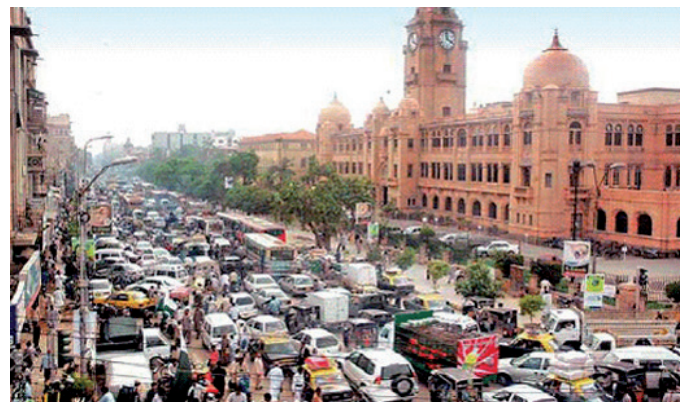

Fotografía № 1: Embotellamiento en las calles de Karachi. en: http://http://farm2.static.flickr. com/1318/1112106432_02e032b85b. jpg (último acceso: Mayo 11, 2010). 
casos de gestión de tráfico en ciudades hermanas donde se han alcanzado resultados exitosos.

Existen algunas buenas prácticas a nivel mundial que tienen como objetivo dar la preferencia a los peatones en lugar del coche, tal es el caso de Londres, donde se cargan impuestos de congestión a los vehículos que entran en el centro de la ciudad, logrando de esta manera, reducir el ingreso de vehículos a la misma ${ }^{4}$. París es otro caso de aplicación efectiva de políticas de manejo del tráfico, donde se ha implantado en los últimos años un proyecto de ampliación de aceras y creación de carriles de bicicleta, además de la construcción de carriles exclusivos para buses a lo largo de algunas avenidas importantes ${ }^{5}$.

El propósito principal es calmar el tráfico sin afectar el transporte público. En este sentido, hay varias acciones que pueden ser tomadas en cuenta como las sugeridas por la Union Internationale des Transports Publics, que plantea que con la reducción de la velocidad en la zona a $30 \mathrm{~km} / \mathrm{h}$, la contaminación del aire y los niveles de ruido disminuyen enormemente. Esta medida puede aplicarse conjuntamente con el uso de obstáculos horizontales que reducen la velocidad, tales como cojines de velocidad, jorobas sinusoidales, montículos de meseta, etcétera ${ }^{6}$.

Es conveniente establecer un sistema de gestión completo, tal como se ha hecho en Brujas y el Vieux Quebec. Ambos centros históricos Patrimonio Mundial serán analizados como posibles ejemplos de las políticas de gestión que pueden ser transferibles a Cuenca, teniendo en cuenta que ambas son ciudades de tamaño medio y contenedoras de valores patrimoniales. La situación de Quebec es muy interesante en relación al caso de Cuenca, teniendo en cuenta las características topográficas de su centro histórico.

\section{A. Brujas}

La ciudad medieval de Brujas (Bélgica) tiene una superficie de 410 hectáreas, donde viven alrededor de veinte mil personas. En los últimos treinta años ha habido un aumento considerable de las obras de restauración y renovación en el centro histórico. "La ciudad fue concebida originalmente para los peatones, con pequeñas tiendas y casas comerciales, y no para las grandes empresas industriales o comerciales. Los viejos edificios cada vez estuvieron más amenazados por el tráfico, el elevado desarrollo comercial..."”

A finales de los años sesenta, una de cada ocho casas estaba vacía o abandonada en el centro de la ciudad de Brujas, y muchos de estos edificios se transformaron en conventillos, con el consecuente daño para el patrimonio construido.

(Ver Fotografía $\mathrm{N}^{\circ}$. 2)
En el año 1972 se crea un plan de gestión para mejorar la habitabilidad y las condiciones de conservación del patrimonio en la vieja ciudad, con la finalidad de eliminar el ruido y la contaminación, y promover el regreso de los habitantes de la ciudad ${ }^{8}$.

El Gobierno local se percató de la necesidad de crear una política de tráfico con la intención de dar preferencia a los peatones sobre los coches. Así, la ciudad fue dividida en nueve partes conectadas por corredores que tenían una forma de bucle, cada uno de estos corredores se iniciaba en el anillo perimetral, pasaba a través de la ciudad y terminaban en el punto de partida. Mediante la creación de dichos bucles se dio una disminución del tránsito. Se daba a los habitantes la posibilidad de acceder a lugares de estacionamiento cerca de sus casas, conjuntamente con la creación de varios estacionamientos de borde para los visitantes.

Con la implementación de estas políticas, la ciudad se convirtió en un lugar más agradable para vivir, se renovaron varias plazas públicas y la antigua plaza del mercado se bloqueó al tránsito de los coches privados para fomentar el transporte público y de peatones combinado con el tránsito de bicicleta. (Ver Fotografía $\mathrm{N}^{\circ}$. 3)

Se incentivó el uso de la bicicleta, permitiendo que éstas puedan acceder a casi todos los puntos de la ciudad (excepto en las zonas totalmente peatonalizadas), incluyendo plazas y calles donde los vehículos están prohibidos.

También se ha mejorado el transporte público de Brujas con la ayuda de la empresa de transporte público local. La flota de autobuses fue renovada mediante la implementación de unidades más pequeñas y maniobrables, autobuses amigables con el medio ambiente y más accesibles.

Dado que la mayoría de los edificios de la ciudad carecía de garajes de estacionamiento, se construyó en varias calles una zona azul de parqueo a corto plazo. Para un estacionamiento a largo plazo se construyeron varios parqueaderos públicos en la ciudad. Los habitantes tenían preferencia y acceso a suscripciones baratas mediante la presentación de su tarjeta de residencia.

Algunos aparcamientos de borde fueron construidos con la finalidad de evitar la entrada de gran cantidad de coches pertenecientes a los visitantes de la ciudad vieja. Estos aparcamientos están muy bien conectados con el servicio de autobuses, para de esta manera acceder fácilmente al centro de la ciudad.

Con el objetivo de alcanzar las normas ambientales europeas, la ciudad de Brujas y De Lijn (la compañía de buses local) han puesto en marcha desde el 2009 un autobús híbrido. Esta es una opción menos ruidosa y más limpia que otros tipos de bus. La idea es cuidar de la salud de sus habitantes, así como el medio ambiente, el patrimonio natural y el construido. 
En el año 2010 se planteó la implementación de cuatro autobuses híbridos, que serán los primeros de un reemplazo total de la flota de autobuses a un tipo más amigable del medio ambiente 9 .

Como resultado de la aplicación de un sistema completo de gestión del tráfico, hoy en día:

La amenaza de los vehículos motorizados para el patrimonio histórico es menos aguda, debido a la reducción de los vapores de la gasolina y la vibración de edificaciones. La contaminación atmosférica es un problema general, no específico de Brujas, pero la ciudad tiene la ventaja de no tener una industria muy contaminante en sus inmediaciones ${ }^{10}$.

\section{B. Vieux Quebec}

El barrio conocido como Vieux Quebec (Viejo Quebec) ha sido incluido en la lista del Patrimonio Mundial a partir de 1985. Está compuesto por 135 hectáreas, donde viven alrededor de 5750 personas (censo 2001). La ciudad de Quebec (Canadá) es el primer asentamiento colonial de América del Norte, fundada alrededor de 1609 en la llegada de los conquistadores franceses. La ubicación de la ciudad fue escogida por razones de estrategia militar, aprovechando la topografía natural. Hay un gran desnivel entre la Haute-Ville (ciudad alta) y la Basse-Ville (ciudad baja) que limita la movilidad dentro de la ciudad vieja. (Ver Fotografía $\mathrm{N}^{\circ}$. 4)

Desde los primeros años del siglo XX, la implantación de un tranvía promovió que la población trasladara su vivienda fuera de la ciudad y siga trabajando en ella, con lo que se generó un éxodo hacia los suburbios.

En la década de 1950, la universidad local (Universidad Laval) se trasladó fuera del centro de la ciudad a un campus más espacioso, generando también la despoblación de algunas zonas del centro porque muchos estudiantes -que se encontraban en ese tiempo alojados en viviendas asequibles dentro de la Vieja Ciudad- decidieron buscar nuevos lugares cerca de la trasladada universidad. Hubo un decrecimiento poblacional en la Vieja Ciudad desde la década de 1960 hasta 1996, año en el que la curva empezó a revertirse como resultado de los planes de revitalización.

El transporte público y la accesibilidad desempeñan un papel muy importante en la vitalidad de una ciudad histórica, así como en la atracción y mantenimiento de sus habitantes. Sin embargo, teniendo en cuenta las estrechas calles de la vieja ciudad, sumado al delicado equilibrio de la calidad ambiental, la Municipalidad tuvo que tomar varias medidas para su gestión con el fin de reducir los gases de efecto invernadero y el ruido producido por el transporte.

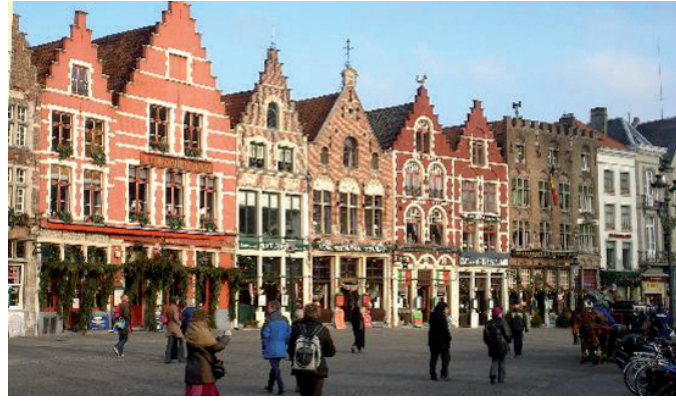

Fotografía № 2 La plaza central de Brujas, (peatonalizada), en: http://media-cdn tripadvisor.com/media/photos/01/0a/55/b1/grote-markt-brugge-belgium.jpg (último acceso: Mayo 11, 2010)

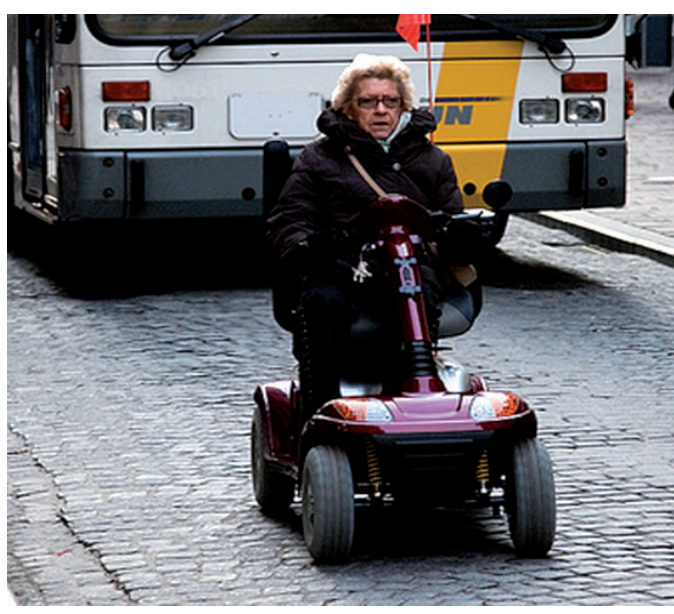

Fotografía № 3: La ciudad patrimonial da preferencia a la persona por sobre cualquier otro medio de transporte. En: http://www.flickr.com/ photos/pjmeade/4059751719/ (último accesso: Mayo 11, 2010).

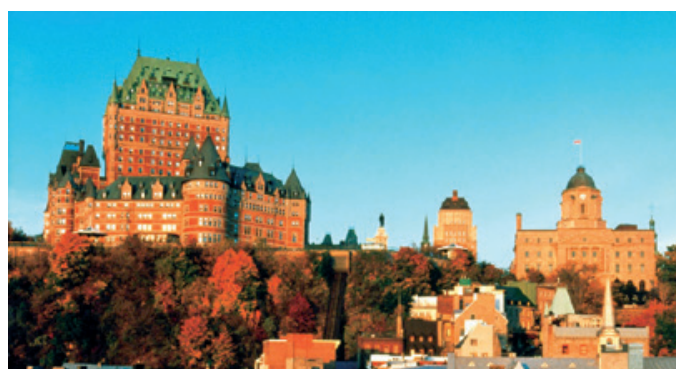

Fotografía № 4: El Vieux Quebec, una ciudad patrimonial ubicada en lo alto de una colina, con problemas obvios de accesibilidad. En http://www.canadatravelsolution.com/gallery/quebecoldcity.jpg(último acceso: Mayo 5, 2010) 
A los coches particulares se les permite el paso por la ciudad histórica, pero como una manera de desincentivarlo cada vez se eleva más el costo del estacionamiento en su interior. Esta estrategia ha sido adoptada para fomentar la peatonalización de la zona y el uso del transporte público.

Como medida complementaria se han creado varios estacionamientos de borde en la parte

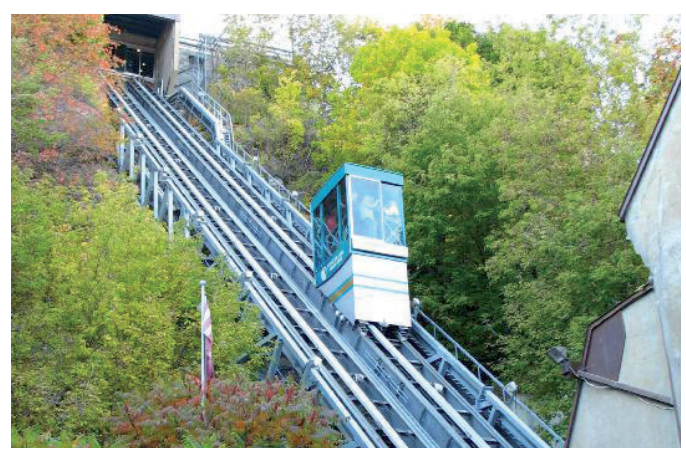

Fotografía № 5: Funicular que conecta la ciudad alta con la baja, transporte gratuito para las personas que poseen una tarjeta de transporte público. En: http://image18.webshots.com /18/4/93/3/27974930300549357 57shlHwC_ph.jpg (último acceso: Mayo 05, 2010). baja de la ciudad, estas plazas de aparcamiento están bien servidas por el sistema de transporte público; existe una política de transporte público gratuito en el interior del centro de la ciudad. Además, para ayudar a la gente a sortear el desnivel topográfico de acceder a la ciudad a pie, se otorgan viajes gratuitos en el funicular para las personas que posean una tarjeta de transporte público ${ }^{12}$. (Ver Fotografía $\mathrm{N}^{\circ} .5$ )

A partir del año 2007 se pusieron a prueba nuevos microbuses ecológicos eléctricos, buscando mejorar el transporte público, éstos tienen por objetivo ser un medio de transporte de alta frecuencia, a pesar de su baja capacidad, que ayudaría a evitar la contaminación, siendo gratuitos dentro de la vieja ciudad.

Se han creado lugares exclusivos para peatones y ciclistas con el nombre de "zones d'ambience". El uso de la bicicleta dentro de la ciudad histórica se ha motivado generando varias zonas de aparcamiento seguras y ascensores para bicicletas, así como carriles cerca de las escaleras para mejorar la accesibilidad y superar el problema topográfico. En los autobuses urbanos se implementaron soportes para bicicleta fomentando la unión flexible de la bicicleta y el transporte público.

Como resultado de dichas medidas hubo una reducción importante de los contaminantes y el ruido en el centro histórico. En consecuencia, esto ha generado una mejora de la calidad de vida de sus habitantes.

\begin{tabular}{|c|c|c|}
\hline POLÍTICAS & BRUJAS & VIEUX QUEBEC \\
\hline $\begin{array}{l}\text { ACCESIBILIDAD } \\
\text { DE COCHES }\end{array}$ & $\begin{array}{l}\text { Bucles } \\
\text { Barreras } \\
\text { Plaza central inaccesible } \\
\text { Horarios para aprovisionamiento de mercadería y materias primas }\end{array}$ & \\
\hline PARQUEADEROS & $\begin{array}{l}\text { Implementación de parqueaderos de borde } \\
\text { Política de reducción de puestos de parqueo dentro del centro histórico } \\
\text { Subvención de parqueo para residentes }\end{array}$ & \\
\hline PEATONALIZACIÓN & $\begin{array}{l}\text { Áreas totalmente peatonales, donde ni siquiera } \\
\text { las bicicletas están permitidas }\end{array}$ & $\begin{array}{l}\text { Peatonalización de varias áreas creando } \\
\text { "Zones d'Ambience" }\end{array}$ \\
\hline BICICLETAS & $\begin{array}{l}\text { Política de fomento del uso de bicicletas } \\
\text { Lugares para estacionamiento de bicicletas }\end{array}$ & $\begin{array}{l}\text { Política de fomento del uso de bicicletas } \\
\text { Lugares para estacionamiento de bicicletas } \\
\text { Ascensor para bicicletas y rieles para superar } \\
\text { los problemas topográficos }\end{array}$ \\
\hline $\begin{array}{l}\text { TRANSPORTE } \\
\text { PÚBLICO }\end{array}$ & $\begin{array}{l}\text { Sistema de transporte mediante autobuses } \\
\text { Minibus ecológico de alta frecuencia }\end{array}$ & $\begin{array}{l}\text { Transporte gratuito mediante bus en el centro histórico } \\
\text { Pasaje de funicular gratis para personas que tienen } \\
\text { una tarjeta de transporte público }\end{array}$ \\
\hline $\begin{array}{l}\text { CONSERVACIÓN DEL } \\
\text { PATRIMONIOEDIFICADO }\end{array}$ & \multicolumn{2}{|c|}{ Conservación de edificaciones patrimoniales para onvertirlas en vivienda asequibles } \\
\hline
\end{tabular}




\section{Tecnologías para los sistemas de gestión de tráfico}

Una de las decisiones básicas a ser adoptadas para controlar el tráfico en las ciudades patrimoniales está relacionada con el control del acceso de vehículos privados y el fomento de un sistema de transporte masivo adecuado. Ese objetivo debe contribuir a mantener viva la zona e incluso animar a los ciudadanos para repoblar las ciudades históricas que se encuentran abandonadas.

En este sentido, sería conveniente conocer en detalle las ventajas y desventajas de los sistemas de transportación masiva más conocidos, esto es, de los sistemas de tranvía y autobús.

\section{Sistema de tranvía vs. Sistema de au- tobús}

Hasta la primera mitad del siglo XX muchas ciudades europeas fueron atendidas por sistemas de tranvía. Sin embargo, en los años sesenta, la mayoría de ellos desaparecieron para dejar espacio a la circulación de automotores, porque se creía que los autobuses tenían la flexibilidad para proporcionar un mejor servicio. Cuando los niveles de contaminación aumentaron drásticamente, muchas ciudades tomaron la decisión de volver al tranvía. ${ }^{13}$

Según Gunnarson y Lofgren (2001), la energía necesaria para la ejecución de un sistema de tranvía es menor por persona $/ \mathrm{km}$ que la que necesitan los coches, lo que le convierte en una mejor alternativa. Los tranvías funcionan con electricidad en lugar de gasolina, lo que implica una reducción de las emisiones de $\mathrm{CO} 2$ y del ruido. También resultan eficientes en términos de espacio, un factor muy importante si se pretende implementarlo en las calles de ciudades históricas que en su mayoría son más bien estrechas. Empero, una de las desventajas del tranvía es que no puede seguir rutas sinuosas ya que opera sobre rieles y necesita un amplio radio de giro.

Por otro lado, los sistemas de autobús tienen la ventaja de los bajos costos de implementación y el hecho de que son fácilmente accesibles en las ciudades pequeñas y medianas, a diferencia del tranvía, cuya inversión inicial es muy alta. La desventaja de un sistema de buses radica en la facilidad de ser desregulado, porque puede ser afectado por el tránsito ${ }^{14}$. Otro problema es que el sistema de buses por lo general no cuenta con una muy alta estima entre la población que se moviliza mediante automotores privados, y seguramente lo pensarían dos veces antes de dejar sus coches para desplazarse en autobús (Edwards y Mackett 1996).

Una opción adecuada es la creación de sistemas integrados, donde los autobuses alimentan un tranvía, tal es el caso de algunas ciudades de América del Norte y Europa como Vancouver, Miami, Gante, etcétera.

\begin{tabular}{|c|c|}
\hline A FAVOR DEL TRANVÍA & EN CONTRA DEL TRANVÍA \\
\hline $\begin{array}{l}\text { Imagen: } \\
\text { - Se percibe como un elemento que atraería a los conductores } \\
\text { - Se percibe como un elemento que estimularía el desarrollo } \\
\text { - La infraestructura fija denota permanencia }\end{array}$ & $\begin{array}{l}\text { - Alto costo de inversión que restringe el crecimiento de la red por } \\
\text { una suma dada de dinero }\end{array}$ \\
\hline $\begin{array}{l}\text { - No causa contaminación en las vías } \\
\text { - Puede funcionar sobre la calle o sobre una riel }\end{array}$ & - Ruta inflexible [no puede ser alterada una vez construida] \\
\hline A FAVOR DEL BUS & EN CONTRA DEL BUS \\
\hline $\begin{array}{l}\text { - Bajo costo que permite incrementar la red por } \\
\text { una suma dada de dinero }\end{array}$ & $\begin{array}{l}\text { Imagen: } \\
\text { - Se percibe como un elemento que no atraería a los conductores } \\
\text { - Se percibe como un elemento que no estimularía el desarrollo } \\
\text { - No se le considera un servicio permanente }\end{array}$ \\
\hline - Rutas flexibles & - Dificultad de obtener financiamiento \\
\hline & - Causa contaminación en las vías \\
\hline
\end{tabular}




\section{El problema del tráfico en Cuenca:}

La propiedad de vehículos motorizados ha ido aumentando paulatinamente en algunas naciones de América Latina, debido en parte a los altos ingresos generados por la exportación petrolera en las últimas cuatro décadas. Cuenca, siendo la tercera ciudad más grande del Ecuador, con una población de alrededor de 400000 habitantes $^{15}$, ha sufrido un aumento considerable en el tránsito. De hecho, hoy en día, Cuenca es una de las ciudades del Ecuador con la tasa más alta de propietarios de automóviles per cápita: un coche por cada cuatro personas (Bleviss, 2000: 50). De acuerdo con la información más reciente hay alrededor de 74000 vehículos $^{16}$ que circulan por la ciudad, y una gran cantidad de ellos diariamente van, o al menos pasan, por el centro histórico. La cantidad de vehículos ha seguido aumentando en los últimos años; por citar un ejemplo, en el año 2000 había alrededor de $38000^{17}$ coches en la ciudad, que en aquel entonces ya era considerado como problemático. Hoy en día se puede asegurar que está fuera de control.

\section{Deficiente sistema de transporte público}

Posiblemente el aumento de automóviles privados en la ciudad podría estar relacionado con el hecho de que el servicio de transporte público es muy deficiente. Los autobuses actualmente son manejados por empresas privadas, no tienen horarios organizados, por lo que la gente no puede confiar plenamente en ellos. Por otra parte, ir en autobús alrededor de la ciudad no es una forma de transporte muy segura, ya que hay un alto nivel de robos en sus unidades. Adicionalmente, un número notable de accidentes de tránsito involucran a autobuses, ya que sus conductores son bien conocidos por su falta de respeto de las normas de tránsito.

El problema del transporte público en la ciudad no es nuevo. Originalmente, la responsabilidad sobre la gestión del transporte estaba en manos del Gobierno nacional y en 1999 se transmitieron las competencias a la Municipalidad (Bleviss, 2000: 51). Antes de que se lleve a cabo esta transición, la calidad del transporte público era aún más deficiente. La ciudad estaba servida por unidades de bus de por lo menos 18 años de edad, y por supuesto, más contaminantes. Desde entonces, el Municipio ha creado las normas reglamentarias e instrucciones para controlar este problema ${ }^{18}$. Felizmente, la flota de autobuses se ha ido renovando y nuevas unidades más respetuosas con el medio ambiente son las que circulan en la actualidad. Sin embargo, la mayoría de ellas siguen utilizando diesel como combustible, por lo que todavía son contaminantes.

Además del tránsito urbano circulando por la ciudad, hasta el año 2007 los buses interprovinciales podían pasar a través del Centro Histórico ${ }^{19}$. Hoy en día, los autobuses urbanos en Cuenca circulan por casi todas las calles de la ciudad histórica, generando contaminación, vibraciones y ruido. Hace pocos años la Municipalidad prohibió su entrada al Parque Calderón y organizó canales exclusivos para la circulación de autobuses en dirección este-oeste por los límites exteriores de la histórica ciudad, aunque sin mucho éxito.

\section{La circulación excesiva de vehículos motorizados privados dentro de la ciudad histórica}

Lamentablemente, los vehículos particulares pueden circular por todo el Centro Histórico; literalmente la ciudad ha sido entregada a los conductores de automóviles en detrimento de los peatones. Se evidencian conflictos entre los vehículos y los caminantes: los cruces peatonales (cuando están señalizados) no son respetados por los automovilistas y los peatones no tienen ninguna zona exclusiva en el Centro Histórico o en el resto de la ciudad.

Lo antedicho se ve complicado debido a que, por lo general, los ciudadanos evitan caminar desde sus casas a sus lugares de trabajo y por el Centro Histórico, aunque la ciudad sigue siendo muy pequeña y aún es posible moverse sin coche. Es cierto que la topografía de Cuenca, con sus grandes pendientes, dificulta la accesibilidad; se ha buscado alentar la movilidad peatonal mediante la creación de varios puentes exclusivos y escalinatas para lograr una mejor conexión entre la ciudad histórica de las zonas nuevas, no obstante, es necesario reconocer que todavía es muy difícil llegar a la ciudad alta en bicicleta.

Hasta 1999 se permitía a los conductores aparcar sus coches en las estrechas calles de Cuenca de forma gratuita, lo que teniendo en cuenta la gran cantidad de automóviles existentes, generaba aún más congestión. A partir de ese año se implementó un "sistema de estacionamiento rotativo tarifado" dentro de la ciudad para motivar las actividades comerciales y administrativas, ofreciendo a los usuarios un fácil acceso a las zonas de estacionamiento del Centro Histórico (Empresa Municipal de Terminales Terrestres y Estacionamiento Tarifado).

La implementación de esta medida prohíbe el estacionamiento de vehículos en las nueve cuadras alrededor de la plaza central y mejora la movilidad al desalentar que los usuarios dejen sus coches en las calles durante largos períodos de tiempo. Como resultado, la capacidad de varias vías mejoró, pero también provocó una proliferación de las plazas de aparcamiento privado en la zona protegida. En el año 2005 había alrededor de 1600 plazas de estacionamiento público rotativo en las calles del centro histórico y cerca de 700 espacios dentro de propiedades privadas ${ }^{20}$.

En el mismo año, con el apoyo del Proyecto El Barranco, la Municipalidad sugirió la creación de varios aparcamientos subterráneos que podrían ubicarse por debajo de importantes plazas de la ciudad como la Plazoleta de la Cruz del Vado y la Plaza de San Francisco. Estos puntos son muy delicados, están rodeados de patrimonio arquitectónico y son zonas de tránsito conflictivo. Además resulta inconcebible la idea muni- 


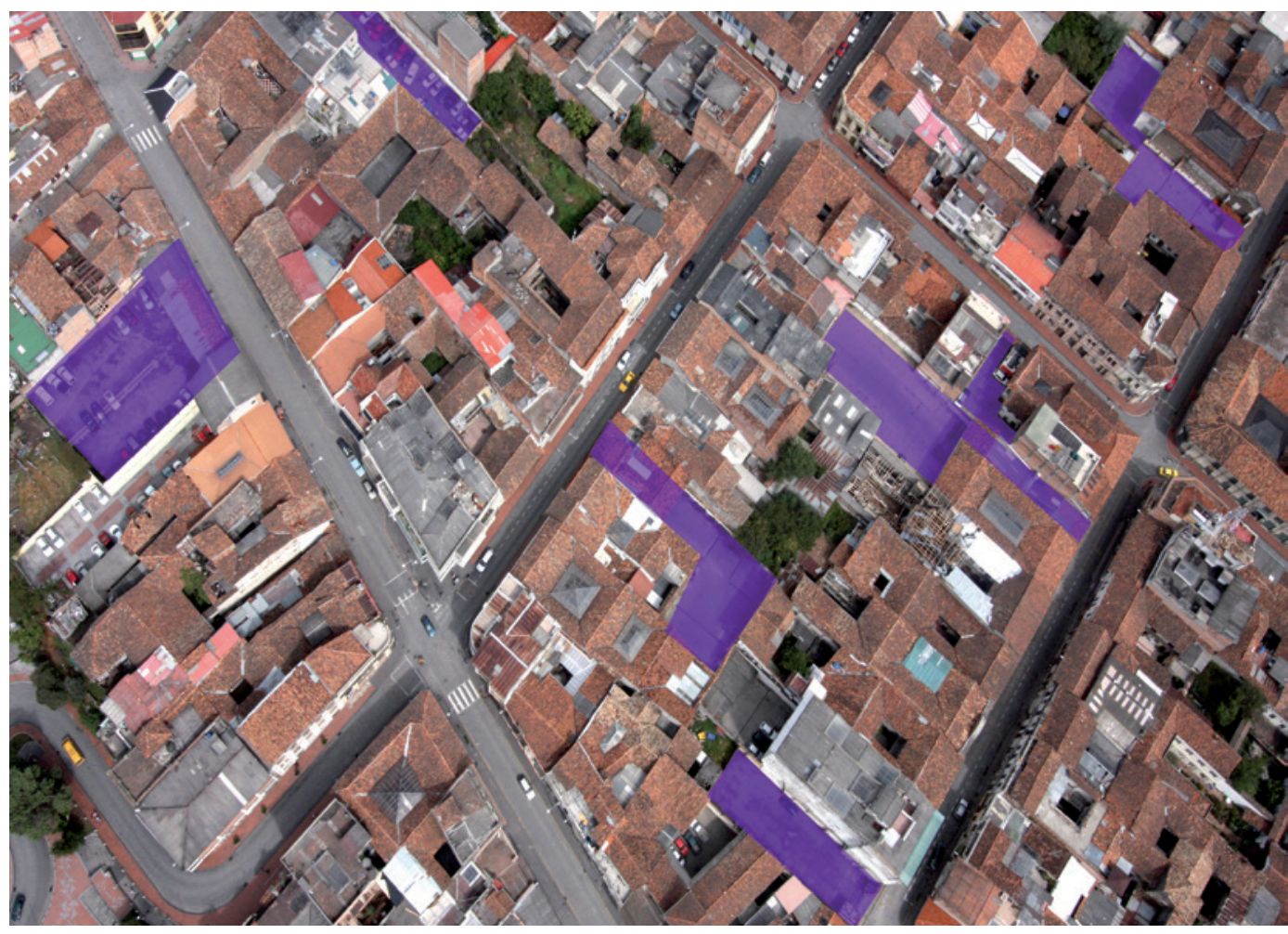

Fotografía № 6: Parqueaderos de la ciudad histórica en corazones de manzanas. Fotografía de Fausto Cardoso Martínez. Noviembre, 2010 (modificada por la autora)

cipal de crear más plazas de aparcamiento en el interior del Centro Histórico, cuando el objetivo es precisamente la reducción de la cantidad de coches que circulan a través de él. Ventajosamente, estas iniciativas no han sido implementadas.

\section{Estacionamientos y fachadismo}

En el Centro Histórico de Cuenca, varios lotes de estacionamiento se han creado dentro de los patios de las viejas casas. Este uso genera la demolición de los interiores de los edificios, quedando sólo la fachada intacta. En algunos casos se ha dado una demolición completa para crear edificios de aparcamiento con la consiguiente afrenta al patrimonio construido ${ }^{21}$.

Para garantizar la seguridad de sus vehículos, los conductores prefieren dejar sus coches en los estacionamientos privados. Por lo tanto, ser dueño de un estacionamiento es un negocio muy rentable para los propietarios de casas antiguas, resultando en la pérdida definitiva de decenas de edificios de valor patrimonial.

En consecuencia, a finales de 1999, la Municipalidad trató de regular la proliferación de estos espacios mediante la creación de la "Ordenanza que regula la implementación de parqueaderos públicos y privados en áreas urbanas de valor histórico", donde se señalaba que:
Los inmuebles destinados a parqueaderos de uso público o privado, podrán implementarse previa autorización expresa de la Comisión de Centro Histórico, en inmuebles que no estén considerados como de valor arquitectónico de Categoría 1 y $2^{22}$.

Esta ordenanza era muy permisiva, acordaba la implementación de estacionamientos dentro de las casas de valor histórico arquitectónico 3. En esta categoría se encuentran casas que carecen de notables características históricas o artísticas, pero que son importantes para la adecuada lectura del contexto y del patrimonio construido de la ciudad ${ }^{23}$.

Este estrecho punto de vista de las autoridades implicó la conservación de tan sólo el bloque frontal de estas casas y la destrucción parcial de edificios históricos que eran estructuras de valor que debían ser preservadas, y donde deberían funcionar usos compatibles con ella. En algunos casos se modificaron también los bloques delanteros de las edificaciones para permitir el acceso de coches, con la consiguiente destrucción de los interiores de la primera planta.

Con fines demostrativos se tomó al azar una muestra del Centro Histórico para estudiar las zonas donde se implementaron los estacionamientos en los centros de manzana. Desde la imagen es evidente que no hubo control municipal sobre la cantidad de plazas de aparcamiento necesarias, ni se tuvo en cuenta la valoración de las edificaciones. (Ver Fotografía $\mathrm{N}^{\circ}$. 6) 
Los corazones de manzana que en un principio fueron utilizados como huertos han desaparecido en gran parte para dejar espacio para los coches, lo que genera aún más contaminación, teniendo en cuenta que esos espacios verdes eran los responsables de la descontaminación del aire; es necesario señalar que no hay muchas zonas verdes en el Centro Histórico destinadas a esta función.

El problema del fachadismo es recurrente no sólo en Cuenca, sino también en muchos centros históricos en todo el mundo. En Bruselas, por ejemplo, en la década de 1960 hubo un proceso de destrucción masiva de la ciudad debido a la especulación de la tierra. La solución encontrada en ese momento fue mantener solamente las fachadas como un método de conservación -aunque muy superficial- de la identidad urbana (Bernal Santa Olalla, 2002: 272). Esta peculiar forma de conservación del patrimonio era conocido como "fachadismo" y desde que se inició en Bruselas se lo conoce también como “bruxellisation" de las ciudades históricas (Hein, 2006: 232).

\section{Las consecuencias generales}

La cantidad de vehículos públicos y privados circulando en la ciudad ha creado varios problemas, uno está relacionado con la contaminación del aire, que ha alcanzado y superado los niveles máximos permitidos de acuerdo a la norma nacional de calidad del aire (Cuencaire, Corporación para el manejo del aire en Cuenca) ${ }^{24}$. La polución no sólo afecta a la salud de los habitantes de Cuenca y la habitabilidad de la ciudad, sino también ha provocado diversas formas de daño físico a los edificios históricos. Las calles del centro de la ciudad de Cuenca son muy estrechas y flanqueadas por construcciones de dos o tres pisos, lo que impide la evacuación de los gases producidos por la combustión del motor. Así, la contaminación se impregna en fachadas de edificios históricos y genera profundas reacciones químicas en muchos materiales de construcción frágiles de dichos inmuebles.

La principal complicación es la cantidad de tiempo que las unidades de transporte público tienen que invertir para recorrer el centro de la ciudad. Debido a la alta congestión, un autobús se tarda aproximadamente 15 minutos para moverse 300 metros, produciéndose una exposición más larga de la ciudad a los gases contaminantes. Este problema va de la mano con la contaminación acústica causada por los coches en el interior del Centro Histórico, afectando a los peatones y causando dificultades de audición.

Adicionalmente, han proliferado los baches en las calles del Centro Histórico causados por el alto nivel de tráfico. El material de calzada es en su mayoría el adoquín colocado sobre material de reposición que ha sido compactado de forma natural con el paso del tiempo. Sin embargo, éste no tiene la resistencia suficiente para soportar el tráfico pesado de vehí- culos públicos y privados. El constante tránsito sobre estas vías deterioradas genera vibraciones, y como resultado de las mismas, grietas en fachadas de edificios históricos.

Por otro lado, los autobuses que dan servicio a la ciudad son fabricados en talleres locales, con cabinas adaptadas a un chasis. El principal problema es la altura del chasis, que produce buses muy altos, no muy convenientes para la ciudad o la accesibilidad de los usuarios, ya que hay que subir cuatro escalones para acceder al interior del autobús. Esta es una cuestión relevante a tener en cuenta cuando se planifica la accesibilidad al sistema de transporte de los usuarios con discapacidad o de edad avanzada.

\section{Soluciones}

A partir de 1999, el Gobierno local de Cuenca ha estado trabajando para mejorar la situación, aunque ha tenido sólo un éxito modesto. La Municipalidad después de una larga negociación y acuerdos con los propietarios y conductores de autobuses ha logrado sustituir las viejas unidades de autobús (aquellas que tenían alrededor de 20 a35 años de edad). Pero los problemas de tráfico están aún muy lejos de ser resueltos porque hasta hoy han sido abordados de una manera muy superficial y política, tratando de favorecer a personas influyentes en lugar de buscar el bienestar de toda la ciudad, sus ciudadanos y el patrimonio construido.

Desde el punto de vista oficial, han surgido varias ideas para mejorar el tráfico dentro de la ciudad histórica. Algunas de ellas procedían de una ideología técnica, muy cerrada, que no respondieron a las necesidades de una ciudad patrimonial. Por ejemplo, en 2001, el gerente de la Unidad Municipal de Tránsito (UMT), sugirió que para mejorar la movilidad en el centro de la ciudad, la eficiencia de los autobuses y evitar la contaminación ambiental, los adoquines debían ser retirados de las calles del Centro Histórico y debían sustituirse por asfalto. Esta fue, evidentemente, una idea que viene de un punto de vista técnico, sin un análisis adecuado de las cualidades patrimoniales que Cuenca debe mantener. Ventajosamente, esta idea nunca se materializó $^{25}$

Una buena práctica fue la regeneración de varias calles y plazas con el fin de dar preferencia a la circulación peatonal sobre el tránsito vehicular mediante la ampliación de las aceras, o hacer más alto el nivel de la calle para crear una plataforma, incluso fomentando en el peatón un sentimiento de pertenencia de los espacios urbanos. Sin embargo fue una respuesta poco comprometida con los peatones, al darles una preferencia a medias. Tal vez esta semipeatonalización se llevó a cabo, hasta ahora, de una manera demasiado condescendiente; no existen zonas exclusivas para el peatón y son la excepción, las pocas plazas donde se les da una cierta prioridad. La peatonalización es una política que debe ser puesta en operación de una manera más generalizada en el Centro Histórico de la ciudad. No obstante, antes de prohibir completamente el coche, debería estar en funcionamiento un buen sistema de transporte. 


\section{Sistema Integrado de Transporte}

Desde el año 2000, la idea de generar un sistema troncal de líneas de autobuses complementada por alimentadores, ya estaba en la mente de las autoridades ${ }^{26}$, pero los estudios previos tomaron mucho tiempo y es sólo a partir de 2007 que se pudo ver que había claridad en la concepción de un Sistema Integrado de Transporte (SIT); a pesar de ello debido a la falta de decisión política, este sistema aún no se ha aplicado plenamente.

Desde el punto de vista oficial, e incluso en las mentes de algunos técnicos, existe la idea de que el transporte público (en concreto el autobús) es el culpable de la contaminación ambiental y de los problemas de tráfico, sin tener en cuenta a los vehículos particulares ${ }^{27}$. La verdad es que los autobuses no son más que la punta del iceberg de la compleja problemática del tráfico, en la que los vehículos privados son también causantes de la contaminación y la congestión, sobre todo si consideramos que gran parte de éstos transportan a una sola persona. Por lo tanto, la solución para los atascos de tráfico, la contaminación atmosférica y el ruido es la implementación de un sistema de transporte adecuado, que anime a los ciudadanos a utilizarlo en lugar de conducir sus coches hacia el Centro Histórico.

En este contexto, la Municipalidad ha diseñado un Sistema Integrado de Transporte que pretende juntar diferentes medios de transporte haciéndolo accesible mediante el pago de una sola tarifa. La primera etapa del sistema está compuesta por tres tipos de líneas de bus:

- Líneas troncales: pasan por el Centro Histórico por una ruta seleccionada en el sentido noreste-sureste y viceversa, pasando por dos estaciones de transferencia: Terminal Terrestre y Feria Libre (ver imagen No. 9).

- Líneas alimentadoras: alimentan el sistema integrado de ida y vuelta de los pueblos cercanos.

- Líneas de anillo: van por los límites del Centro Histórico. Se conectan con los terminales.

Con la implementación del sistema, la mayoría de las unidades de autobuses no pasarían más por el Centro Histórico, solucionando en parte el problema de tránsito existente. El centro estará mejor servido por una línea de transporte que lo conectará con el resto de la ciudad y que permitirá a sus habitantes llevar a cabo las actividades de una manera más organizada, ya que habrá paradas y horarios establecidos.

La segunda etapa uniría dos estaciones más: Norte y Sur, una en las intersecciones de la Panamericana Norte y la Avenida de las Américas (zona del Parque Industrial) y la otra en la intersección de la Avenida de las Américas y la Panamericana Sur. (Ver Gráfico $\mathrm{N}^{\circ}$. 1)

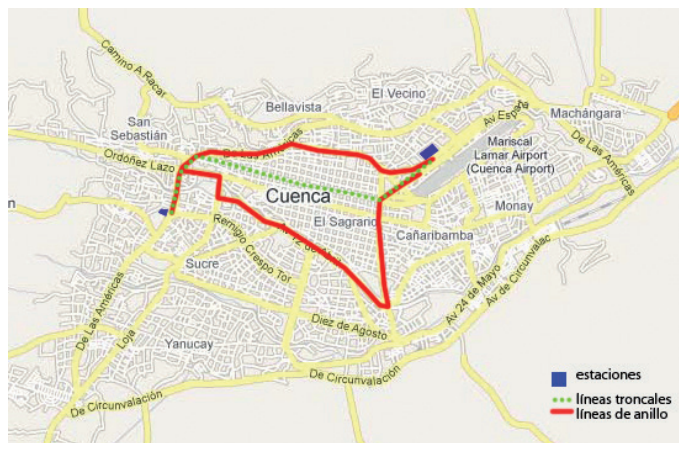

Gráfico № 1: Sistema Integrado de Transportes, Fase I. Esquema dibujado por el autor en base a la descripción. En Ilustre Municipalidad de Cuenca, , "Sistema integrado de transporte de Cuenca" (UMT). http://www.cuenca.gov.ec/ sit/index.html (último acceso: Noviembre 23, 2009). 
Se había planificado que la primera etapa estaría funcionando a partir de mediados de 2007. En la actualidad, por supuesto, todavía no se ha implementado, debido a la negociación para la fusión de todas las empresas de autobuses existentes en tres consorcios y la falta de decisión política.

De acuerdo con el Plan del 2005, las líneas troncales debían ser implementadas utilizando autobuses articulados (Bus tipo 2), cada uno con una capacidad de cien pasajeros. Mientras que las líneas alimentadoras deben utilizar los ya existentes buses "ecológicos" que hoy en día recorren la ciudad. No obstante, desde el final de la pasada administración de la ciudad en 2009, el alcalde electo ofreció durante la campaña que implementaría un tranvía. Por lo que en la actualidad la idea de la Bus tipo 2 ha sido cambiada a un sistema de tranvías. Todavía no se ha definido la posibilidad real de llevar a cabo este plan, ni los costos de implementación, el mismo se encuentra en un estado de prefactibilidad que incluye varios estudios arqueológicos en las calles por donde el tranvía posiblemente pasaría.

Es importante enfatizar que los sistemas de bus son siempre más baratos y más flexibles que los de tranvías, pero su aplicación sigue dependiendo de la sinuosidad de la ruta que las líneas troncales tomarían. La atracción de los usuarios hacia el tranvía es un punto a su favor. Lo ideal sería usar un sistema amigable con el medio ambiente y el patrimonio; seguramente los microbuses híbridos no son la solución para un centro histórico como el de Cuenca, debido a su alto costo y baja capacidad ${ }^{28}$. Es imprescindible que dicho sistema se instale conjuntamente con otras medidas para finalmente resolver el problema del tráfico. Estas medidas deben ser creadas por la Municipalidad y favorables para el patrimonio y el medio ambiente.

\section{Políticas de Gestión}

Es indispensable que Cuenca cuente con un sistema de transporte adecuado, pero sólo tendrá buenos resultados si se complementa con buenas políticas de gestión. Una vez que el sistema de transporte, en base a autobús o tranvía sea implantado, será necesario generar incentivos para que los conductores de automóviles dejen sus vehículos y empiecen a usar el transporte público. Solamente en el momento en que la ciudad esté bien servida por un sistema de transporte adecuado será posible una semipeatonalización del Centro Histórico. Para lograrlo, el Municipio deberá restringir el acceso vehicular al centro mediante la implementación de un sistema de "bucle" ${ }^{29}$, de esta manera, se garantizaría el acceso para la entrega de los bienes de comercio y la accesibilidad para los residentes de la zona; y el tráfico de paso estaría desalentado debido al tiempo y al esfuerzo que los conductores tendrían que invertir para llegar a sus destinos.

Algunos de los estacionamientos existentes deben ser redireccionados para albergar los coches de los residentes, mientras que el resto de ellos deberían desaparecer para convertirse en espacios verdes, cumpliendo su función original como corazones de manzanas $^{30}$.

El Municipio debería asegurar una gran cantidad de estacionamientos de borde fuera de la zona patrimonial, tal como se hace en la ciudad de Quebec. Lo ideal sería diseñarlos de tal manera que no sean muy visibles, para evitar que éstos deterioren el paisaje de espacios tan frágiles como el Barranco. Deben estar conectados al sistema integrado de transporte para garantizar que los conductores puedan acceder fácilmente al Centro Histórico, además de mejorar la accesibilidad a pie, evitando barreras arquitectónicas.

Para fomentar el uso de la bicicleta dentro de la ciudad histórica, es necesario implementar varias plazas de aparcamiento en las plazoletas de la ciudad, de una manera estética. La colocación de rieles en las escaleras existentes, que conectan la parte baja de la ciudad alta, ayudaría a superar fácilmente el problema topográfico que representa un esfuerzo para los ciclistas. Debido a las limitaciones económicas de nuestra ciudad, consideramos que no sería posible crear ascensores para bicicletas como los existentes en Québec, pero los rieles son una buena opción para resolver este problema.

Todas estas políticas podrían mejorar la habitabilidad del Centro Histórico de Cuenca, así se fomentaría el uso de vivienda, lo que garantizaría una mejor conservación del patrimonio construido, ya que es evidente que el uso adecuado es la mejor manera de mantener un bien patrimonial.

\section{Conclusiones}

En el curso de la investigación se comprobó que el tránsito es un problema muy complejo que no sólo afecta a la estructura física de los edificios y calles sino también influye en la habitabilidad de la ciudad histórica y sus valores inmateriales que finalmente derivarían en el despoblamiento de la misma y en problemas de conservación.

Para resolver estos inconvenientes es necesario formular un plan de gestión integral del tránsito, de tal manera que al implementar el sistema de transporte que mejor se ajuste a la ciudad patrimonio, se puedan generar medidas para desalentar el acceso de ve- 
hículos motorizados privados a la ciudad histórica y fomentar la peatonalización, así como el uso de la bicicleta como medio de transporte amigable con el medio ambiente y el patrimonio.

Cuenca, siendo una ciudad Patrimonio de la Humanidad está muy atrasada con respecto a sus homólogas en materia de gestión del tráfico. Brujas, que logró su inclusión en la lista del Patrimonio Mundial de la UNESCO un año después de Cuenca, ha tomado en serio el problema del tráfico y ha implementado medidas para cuidar sus valores patrimoniales y aumentar la calidad de vida en la ciudad.

Cuenca lleva más de diez años discutiendo la implementación de un plan de gestión del transporte y del tránsito, debido a la falta de políticas como la peatonalización del Centro Histórico, por ser una decisión impopular de alto costo político.

La prioridad que tienen los coches en el sistema de transporte de Cuenca, a más de causar complicaciones ambientales, ha provocado grandes problemas de conservación en la ciudad histórica, no sólo relacionados con los daños estéticos en las fachadas de edificios patrimoniales producidos por el hollín, sino con la destrucción de los interiores de muchas casas para crear estacionamientos. Esta situación también ha agravado el problema de la contaminación del aire debido a la pérdida de áreas verdes dentro de las manzanas.

Los centros históricos no estaban diseñados para soportar el pesado tráfico de vehículos motorizados; así, la mejor política sería volver a la forma original de transporte, peatonalizándolos, con el apoyo de un sistema integrado de transporte que sea amigable con el patrimonio y el medio ambiente. Las ciudades históricas deben ser vividas y experimentadas por las personas para ser valoradas y conservadas.

Conservar las características históricas de la ciudad y el medio ambiente no es sólo tarea de la Municipalidad, sino también de los ciudadanos de Cuenca, quienes deben hacer conciencia de la necesidad de dejar de lado el coche cuando se desplacen por la ciudad histórica. Esto puede causar algún inconveniente al principio, pero a largo plazo generará bienestar para las personas que trabajan y viven en ella. 


\section{Bibliografía}

El presente artículo fue escrito en mayo del 2010 como parte de los trabajos del programa Master in Conservation of Monuments and $\mathrm{Si}-$ tes of the RLICC, KU Leuven, Bélgica.

Tulia Esther, 2003. "Patología de la piedra por acción de humedades y suciedad" citado por Martínez de V., Beatriz y María del Pilar Aguilar. "Movilidad, ambiente y centros históricos: una reflexión a propósito del sector de la Candelaria, Bogotá D.C., Colombia". Gestion y Ambiente (Universidad Nacional de Colombia). 10, No. 3 (Diciembre, 2007), pp.119-131.

${ }^{3}$ Rai, Ruprama. "Threats to the Spirit of the Place: Urban Space and Squares, Historic Core, Kathmandu". International Icomos, 16th General Assambly. Septiembre-Octubre, 2009. http://www.international.icomos.org/quebec2008/cd/toindex/80_pdf/80-FAsv-73.pdf (último acceso: Febrero 12, 2010).

${ }^{4}$ Ethan Kent. "Finding a Place for Parking" Making Places. Junio, 2005. http://www.pps.ort/ info/newsletter/june2005/place_for_parking (último acceso: Marzo 5, 2010).

Juliette Michaelson, -Lessons from Paris.- Making Places. Junio 2005. http://www.pps.org/ paris-2/

(último acceso: Marzo 5, 2010).

6 "Traffic calming measures and bus traffic". Union Internationale des Transports Publics. Agosto, 2002. www.uitp.org/Public-Transport/ urban/index.cfm (último acceso: Marzo 5, 2010).

Brigitte Beernaert y Werner Desimpelaere. "Bruges, Belgium". En Management of Historic Centre, editado por Robert Pickard, 8-30. Londres: Spon Press, 2001. p.10.

${ }^{8}$ Brigitte Beernaert y Werner Desimpelaere, op.cit., p.21.

9 Moenaert, Patrick. "Toespraak: Persvoorsteeling nieuwe hybride citybus". Brujas, 27 de abril de 2009. http://www.brugge.be/internet/nl/Mobiliteit/index.htm (último acceso: Marzo 21, 2010).

${ }^{10}$ Brigitte Beernaert y Werner Desimpelaere, op.cit., p. 22
${ }^{11}$ "Plan Directeur du Vieux Quebec". Ville de Quebec. Junio, 2008 http://www.ville.quebec.qc.ca/apropos/vie_democratique/ participation_citoyenne/conseils_quartier/vieuxquebec/docs/ plan_directeur_vieuxquebec_juin2008.pdf (último acceso: Mayo 2, 2010).

${ }^{12}$ Laliberté, Pascal. "Transports viables et amenagement dans le Vieux Quebec: L'heure des choix". Ville de Quebec. Febrero 27, 2006. http://www.ville.quebec.qc.ca/apropos/vie_democratique/participation_citoyenne/conseils_quartier/vieuxquebec/ docs/plan_directeur_vieuxquebec_transport_amenagement. pdf (último acceso: Abril 30, 2010).

La política de transporación pública gratuita ha sido implementada con buenos resultados en otras ciudades como Seattle, Portland y Hasselt. Por ejemplo, en Hasselt la tasa de transporación pública ha aumentado en un estimado de 800 a $900 \%$.

${ }^{13}$ Véase Gunnarsson, Bjorn y Andreas Lofgren. Light Rail-Experiences from Germany, France and Switzerland. Master's Thesis, Lulea: Lulea Teckniska Universitet, 2001; Edwards, Marion y Roger L. Mackett. "Developing new urban public transport systems: An irrational decision-making process". Transport Policy (Elsevier Science Ltd.) 3, No. 4 (1996), pp. 225-239.

${ }^{14}$ Edwards, Marion y Roger L Mackett. "Developing new urban public transport systems: An irrational decision-making process". Transport Policy (Elsevier Science Ltd.) 3, No. 4 (1996): 237.

${ }^{5}$ Esta es la población sin contar con la porción de población flotante que viene de pueblos cercanos a trabajar cada día en la ciudad a comercializar sus bienes en los mercados o a hacer trámites.

${ }^{16}$ Diario El Tiempo. "Contaminacion afecta el Centro Histórico". Abril 16, 2009.

${ }^{17}$ Diario Hoy. "La emisión de gases asfixia el centro de Cuenca". Septiembre 5, 2000.

${ }^{18}$ Cuencaire fue una de las instituciones creadas para controlar los daños ambientales causados por vehículos motorizados. Su responsabilidad es la de generar una evaluación ambiental de cada vehículo de la ciudad una vez al año.

${ }^{19}$ Ilustre Municipalidad de Cuenca. "Proyecto de Ordenanza que regula el tránsito de vehículos de transporte de pasajeros y de carga interprovincial e intercantonal, por las avenidas y pasos laterales de Cuenca". Septiembre 28, 2007. www.cuenca.gov. ec/download/ordenanzas/252_transito.doc (último acceso: Noviembre 22, 2009).

${ }^{20}$ Ilustre Municipalidad de Cuenca. "Proyecto de Ordenanza que regula el tránsito de vehículos de transporte de pasajeros y de carga 
interprovincial e intercantonal, por las avenidas y pasos laterales de Cuenca". Septiembre 28, 2007. www.cuenca.gov.ec/download/ordenanzas/252_transito.doc (último acceso: Noviembre 22, 2009).

${ }^{21}$ Caso del Cine Cuenca.

${ }^{22}$ Concejo Cantonal de Cuenca. "Ordenanza que regula la implementación de parqueaderos públicos y privados en áreas urbanas de valor histórico". Municipalidad de Cuenca. Septiembre 27, 1999. http://www.cuenca.gov.ec/ download/ordenanzas/ordenanza_97.doc (último acceso: Noviembre 23, 2009).

${ }^{23}$ VHIAR1: valor singular y carácter paradigmático. Grado total de protección.

VHIAR2: importantes características arquitectónicas e históricas. Grado parcial de protección.

VHIAR3: sin características históricas, arquitectónicas o artísticas notables, importante para el contexto. Véase: Municipalidad de Cuenca. Catastro Monumental Urbano: actualización, complementación y sistematización del inventario de edificaciones patrimoniales del Centro Histórico. Cuenca, 1999.

${ }^{24}$ Ilustre Municipalidad de Cuenca. Cuencaire, Corporación para el manejo del aire en Cuenca. http://www.cuencaire.org.ec/Cuencaire/ Quienes_somos.faces (último acceso: Noviembre 23, 2009).

${ }^{25}$ Torres, Rodrigo. Director de la Unidad Municipal de Tránsito (UMT), citado en Diario Hoy. "Cuenca, preparan programas contra la contaminación”. Marzo 7, 2001.

${ }^{26}$ Diario Hoy. "La emisión de gases asfixia el centro de Cuenca". Septiembre 5, 2000.

${ }^{27}$ Torres, Rodrigo, Director de la UMT, citado por Diario Hoy. Op. cit.

${ }^{28}$ Ver los casos analizados de Brujas y Vieux Quebec, en este mismo documento.

${ }^{29}$ Tal cual el caso de Brujas, Bélgica.

${ }^{30}$ Idem.

\section{Libros y capítulos de libros}

Amistad, Franklyn T y Jose Regin Regidor. "Traffic Management in a City with U.N. World Heritage Site". Journal of the Eastern Asia Society for Transportation on Studies 6 (2005), pp. 2291-2306.

Bleviss, Deborah. "Urban transportation in Latin America and the Caribbean: the exaple of Cuenca, Ecuador". UNEP Industry and Environment, Octubre-Diciembre 2000, pp. 49-52.

Coronel C., Deifilio. "Cuenca: problemas y soluciones." Revista EI Observador, No. 53 (Octubre, 2009).

Edwards, Marion y Roger L Mackett. "Developing new urban public transport systems: An irrational decision-making process." Transport Policy (Elsevier Science Ltd.) 3, No. 4 (1996), pp. 225 239.

Gunnarsson, Bjorn y Andreas Lofgren. Light Rail-Experiences from Germany, France and Switzerland. Master's Thesis, Lulea: Lulea Teckniska Universitet, 2001.

Gutiérrez Puebla, Javier. "Transporte, movilidad y turismo en los centros historicos". Eria, No. 47 (1998), pp. 241-248.

Hein, Carola. "Brussels-Capital of Belgium and Capital of Europe". en Planning Twentieth Century Capital Cities, David L.A Gordon (ed.), 237-252. New York: Routledge, 2006.

Pickard, Robert (ed), Management of Historic Centres, Londres: Spon Press, 2001.

Tulia Esther, 2003. "Patología de la piedra por acción de humedades y suciedad" citado por Martínez de V., Beatriz y María del Pilar Aguilar. "Movilidad, ambiente y centros históricos: una reflexión a propósito del sector de la Candelaria, Bogotá D.C., Colombia". Gestión y Ambiente (Universidad Nacional de Colombia) 10, No. 3 (Diciembre, 2007), pp. 119-131.

\section{Artículos de Periódicos}

Diario El Tiempo. "El exceso de vehículos causa problemas en el centro." Julio 13, 2009.

Diario El Tiempo. "Empresa municipal de movilidad se creará en abril." Marzo 22, 2010.

Diario Hoy. “¿Se sube Cuenca al Bus?” Noviembre 3, 2005.

Diario Hoy. "Cuenca, preparan programas contra la contaminación.” Marzo 7, 2001.

Diario Hoy. "La emisión de gases asfixia el centro de Cuenca." Septiembre 5, 2000.

Gutiérrez Puebla, Javier. "Transporte, movilidad y turismo en los centros historicos". Eria, No. 47 (1998), pp. 241-248. 